\title{
On Meson Fields and Conformal Transformations
}

\author{
J. A. SCHOUten* \\ Amsterdam University, Holland
}

\section{$\mathrm{T}$} HE invariance of Maxwell's equations for transformations of the orthogonal group in $x, y, z, t$ with 10 parameters having been discovered about 1904 , Cunningham $^{1}$ and Bateman ${ }^{2}$ found in 1910 the invariance for the 15-parametrical group of conformal transformations, containing the orthogonal group. ${ }^{3}$ At that time it was known that conformal transformations in $n$ variables can be dealt with most practically as orthogonal transformations in $n+2$ variables.

Einstein's postulate of universal invariance 'for all natural phenomena applied to the first invariance gave rise to the first or simple theory of relativity and had immediately an enormous success. But as to the second invariance, an application seemed impossible because mechanical phenomena did not fit in any way into a conformal scheme. It was not before 1918 and after the development of general relativity that Weyl succeeded in involving in some way conformal transformations into the theory. His theory was not fully invariant for conformal transformations of the fundamental tensor because his equations also depended on the transformation of an extra geometrical object, that could be identified with the electromagnetic potential vector, but this inconvenience was fully compensated by the fact that the theory gave for the first time a "unification" of gravitation and electromagnetism. This was really something new and very important. Now according to this theory, a bar moving in an electromagnetic field could change its length, and this being not in accordance with experimental facts, it was necessary to make the supposition that the pseudoparallel displacement of the theory had nothing to do with the real movement of a bar. But such an assumption robbed the theory of its most charming features, with the result that notwithstanding its remarkable ingeniosity it retired temporarily to the background. But the idea of unification remained and gave rise to Kaluza's theory of $1921,{ }^{4}$ developed by O. Klein and others. In this theory there were 5 coordinates, and accordingly, there was a tensor of matter and electromagnetism with a matrix with 5 rows and columns in which the fifth one was reserved for electromagnetic quantities. At first physicists felt a bit uneasy about this fifth dimension that could not be accounted for in a reasonable way,

\footnotetext{
* Professor of Mathematics at Amsterdam University: Address : Epe A 126 IX, Netherlands.

1 E. Cunningham, Proc. Lond. Math. Soc. 8, 77 (1910).

${ }^{2}$ H. Bateman, Proc. Lond. Math. Soc. 8, 223, 469 (1910).

${ }^{3}$ In $R_{n}, n>2$, this is the group of point transformations transforming every sphere into a sphere and leaving the origin invariant. $R_{n}=$ flat $n$-dimensional space with a metric defined by a constant fundamental tensor. In an $R_{n}$ there exist lengths and angles and parallel flat subspaces.

${ }_{4}$ Th. Kaluza, Sitz. Preuss. Akad. Wiss. 54, 966 (1921).
}

but in 1930 Veblen and Hoffmann ${ }^{5}$ pointed out that it is sufficient to introduce five coordinates only in the flat local space-time, and that these may be considered as homogeneous projective coordinates. At that time this method was well known from the theory of projective linear connections. What we do in local-time is just what we ordinarily do if we wish, for example, to deal with projective geometry in a plane. This could be done with two ordinary Cartesian coordinates, but this being very clumsy it is much more preferable to use 3 homogeneous coordinates. The main point is that we introduce in local space-time another more general geometry, here projective geometry instead of affine geometry, and it is surprising that this enlarging of the fundamental group enables us to describe physical phenomena from a more general point of view.

Quite independent of these authors, Einstein and Mayer ${ }^{6}$ introduced in 1931 extra 5-dimensional local space-time manifolds. But these spaces are flat metrical spaces with 5 ordinary Cartesian coordinates.

From the projective point of view in every local space-time, the fundamental tensor is represented by a 3-dimensional hypersurface whose equation is quadratic in the 5 local projective coordinates. The use of a different number of coordinates in the local manifolds and in space time in the large can be avoided by using in the large the "curvilinear" homogeneous coordinates, introduced by v. Dantzig" in 1932. In collaboration with v. Dantzig the author applied this method to projective relativity. ${ }^{8}$ Many other authors developed "unitary" theories. They all used 5 coordinates in some way or other. All these theories could be translated into each other fairly well and they succeeded remarkably well as far as unification was concerned. But with respect to the question of the relation between particles and field, culminating into the question of how to avoid an infinite self-energy, they were just as unsuccessful as the fourdimensional theories. As a result, there was an increasina conviction that only quantum mechanics could bring a solution, and the interest in classical ${ }^{9}$ four- and five-dimensional theories began to fade. During the whole process the idea of a conformal theory remained in the background.

Help came for the conformal theory from a quite unexpected side. The spin of the electron, discovered by Uhlenbeck and Goudsmit in 1926, gave rise first to

\footnotetext{
${ }^{5}$ O. Veblen and B. Hoffman, Phys. Rev. 36, 810 (1930).

${ }^{6}$ A. Einstein and W. Mayer, Sitz. Preuss. Akad. Wiss. 25, 541 (1931) ; 26, 130 (1932).

7 D. van Dantzig, Math. Ann. 106, 400 (1932).

${ }^{8}$ See, e.g., J. A. Schouten and D. van Dantzig, Ann. de Math. 34, 271 (1933); Ann. de l'Inst. Poincaré 5, 51 (1935).

"We use the term "classical" in the sense of "non-quantum."
} 
the two-dimensional spin-space of Pauli (spinor analysis of v. d. Waerden) and then to the four-dimensional spin-space of Dirac in 1928. Now what is this spinspace? Consider in an $R_{n}$ the $(n-1)$-dimensional unit sphere with center in the origin. On this sphere there is lying for $n=2 \nu$ one system ${ }^{10}$ of $\infty\left({ }^{\nu+1}{ }_{2}\right) E_{\nu-1}$ 's and for $n=2 \nu+1$ two systems of $\infty\left({ }^{\nu+1}\right) E_{\nu}$ 's. With orthogonal transformations in $R_{n}$ leaving the origin invariant, all these flat spaces and their intersections are transformed into each other and this transformation is called a "representation" of the corresponding orthogonal transformation. Now we may say in a popular way that spin space is nothing else than the world of all these flat spaces on the unit sphere. This assertion, though popular, is quite correct, only it would take some time to make clear in all details the connections between spin-space and the unit sphere, e.g., to prove that spinspace is a flat space, ${ }^{11}$ and that for $n=2 \nu$ its dimension $N$ is equal to $2^{\nu}$. From now on we write $\widetilde{\Im}_{N}$ to denote spin-space. For $n=4$ we get $N=4$ and the vectors in $\widetilde{\subseteq}_{4}$ are the $\infty^{4}$ line vectors ${ }^{12}$ in the $\infty^{3}$ straight lines on the unit sphere ${ }^{13}$ in $R_{4}$.

For $n=6$ there is still another way to get spin-space. Long ago it was known to F. Klein that the $\infty^{4}$ straight lines in 3-dimensional projective space could be considered as the points of a (4-dimensional) quadric in a 5-dimensional projective space (with 6 homogeneous coordinates), or, in another formulation, that the $\infty^{6}$ bivectors $^{14}$ of an $E_{4}$ may be considered as the $\infty^{6}$ vectors of an $R_{6}$ (Plücker-Klein correspondence). Now, about 1930 Veblen got the idea to apply this correspondence to spin theory. Then, the $E_{4}$ is spin space and the 6 coordinates in $R_{6}$ may be considered as the six supernumerary coordinates of a 4-dimensional conformal geometry. The author made the acquaintance with this new idea during his stay in Princeton in 1931 but, however beautiful it might be, nobody seemed to be stimulated to immediate activity and it was not before 1933 that Veblen ${ }^{15}$ and the author, in collaboration with Haantjes ${ }^{16}$ began independently to work out an application. From the beginning it was very striking that the connection between the 4-dimensional spinspace and an $R_{6}$ was in some way primary and that the relations with a 5- or a 4-dimensional space presented itself as secondary cases that could be obtained by specialization.

One thing seemed very queer. We noted that $N=8$ for $n=6$. How does this agree with the $N=4$ that results from the Plücker-Klein correspondence? Orthogo-

\footnotetext{
${ }^{10} E_{n}=n$-dimensional flat affine space. In an $E_{n}$ there exist parallel flat subspaces but no lengths and no angles.

${ }_{11}$ I.e., that coordinates can be chosen in such a way that they transform linearly homogeneously with orthogonal transformations in $R_{n}$.

${ }_{12} \mathrm{~A}$ line vector is a vector which is restricted to lie in a definite straight line. See, e.g., L. Brand, Vector and Tensor Analysis (John Wiley \& Sons, Inc., New York, 1947).

13 J. A. Schouten, Zeits. f. Physik 81, 405 (1933).

${ }^{14}$ Bivector $=$ antisymmetric tensor of valence 2 .

${ }^{15}$ O. Veblen, Proc. Nat. Acad. Sci. 19, 462, 503 (1933).

${ }^{16}$ See reference 13; also, Ann. di Pisa 4, 175 (1935).
}

nal transformations ( $\mathrm{det}= \pm 1$ ) in $R_{n}$ correspond to certain homogeneous linear transformations in $\widetilde{\Xi}_{N}$, but in this $\widetilde{\Im}_{N}$ there exist always two definite $E_{N / 2}$ 's that are individually invariant if $\operatorname{det}=+1$ (rotations) and that are interchanged for det $=-1$ (reflexotations). So, if only rotations in $R_{n}$ are considered, we have two definite $E_{N / 2}$ 's and each of them is transformed into itself. Now for $n=4, N=4$ it can be proved that to every transformation in one $E_{2}$ corresponds just one transformation in the other $E_{2}$ and this means that in this special case we need only one $E_{2}$. This leads to Pauli's representation and v. d. Waerden's spinor analysis. For $n=6$ things are different but next to the ordinary invariants a new invariant emerges ${ }^{17}$ and this happy circumstance allows one of the invariant $E_{4}$ 's to be taken as spin-space. And this spin-space is just the same as the one we arrived at by means of the Plücker-Klein correspondence.

So for the second time and quite unexpectedly conformal geometry made its entrance. Now the general conformal geometry with local conformal space is no longer flat but it has the properties of a quadric in an $(n+1)$ - dimensional flat projective space. In order to avoid the use of two different numbers of coordinates in local spaces and in extended space, the author in collaboration with Haantjes succeeded in 1935 in introducing also $n+2$ homogeneous curvilinear v. Dantzig coordinates in extended space. But here a serious difficulty arose that may be formulated as follows.

If in an ordinary flat projective space of three dimensions an arbitrary surface is considered, we know that the two asymptotic directions at every point of this surface fix in the surface a conformal geometry notwithstanding the fact that in the projective space there is no metric at all. Further, we know that the 4 homogeneous projective coordinates in space can be used as conformal supernumerary coordinates in the surface. Conversely, starting from a 2-dimensional space with a conformal geometry it is always possible to construct a 3-dimensional flat projective space such that the 2dimensional space may be considered as a surface lying in it, and it can be proved that two flat 3-dimensional spaces constructed in this way are "not essentially different," i.e., that they can be mapped on each other in an invariant way. This latter property is very important because otherwise, in order to fix one of these spaces, we would need some additional invariant next to the given conformal geometry.

The same holds for spaces with a dimension $>2$ provided that the conformal geometry is conformalEuclidian. ${ }^{18}$ But this is just the case we are not interested in because it would lead to an "empty" spacetime. Now in $1935-36^{19}$ we proved that to a general

\footnotetext{
17 This invariant will be dealt with in another publication.

18 I.e., transformable into a flat space by means of a conformal transformation.

${ }^{19} \mathrm{~J}$. A. Schouten and J. Haantjes, Proc. Kon. Akad. v. Wet. 38, 706 (1935); 39 (1936); Math. Ann. 112, 594 (1936); 113, 568 (1936).
} 
n-dimensional space with an arbitrary conformal geometry (i.e., a space with a fundamental tensor given to within an arbitrary scalar factor) always an $(n+1)$ dimensional space with a (not flat) projective geometry can be constructed and that two such $(n+1)$-dimensional spaces are "not essentially different," provided that either $n$ be odd or $n$ be equal to 4 and in this latter case, moreover, a special projective tensor of valence 2 (dependent on the derivatives of the fundamental tensor to within the fourth order) be zero. Fortunately, it could be proved that this special tensor vanishes always if the geometry is conformal-Einsteinian, ${ }^{20}$ that is, just in the case that is interesting for physics. This proves that, starting from a conformal and conformalEinsteinian space-time, it is always possible to introduce without ambiguity six homogeneous conformal coordinates in extended space.

After introduction of conformal geometry into physics, the rest-mass is no longer invariant but, instead, a product of rest-mass and length has invariant properties. $^{21}$ That makes clear why relativistic mechanics with its invariant rest-mass did not fit into a conformal scheme. But with this new invariance, Haantjes ${ }^{22}$ could prove in 1940 that conformal transformations are in some way connected with accelerated systems. Hill ${ }^{23}$ hit independently upon the same relation.

All these hints in the direction of a sixth coordinate were so strong that about 1935 it could be predicted that physics would have ere long something to do with the number 6 . But there was still another thing that could have been predicted about that time. In every theory with 6 coordinates there must appear a generalization of the material tensor with a matrix with 6 rows and 6 columns. Now the fifth row and column being already occupied by electromagnetism in the projective theory, the conclusion that probably some unknown physical phenomenon had to exist that could fill the sixth row and column was near at hand and almost obtruding. If some one had hit upon this conclusion, the meson field, predicted by Yukawa ${ }^{24}$ in 1935 could have been predicted from two approaches at the same time. But nobody did, neither in Holland nor, as far as I know, in the United States. History took another course, the conformal theory failed to ask for a meson field, but the meson field came and asked for a conformal theory!

After the prediction of Yukawa and the identification of certain particles of cosmic rays with the particles corresponding to the Yukawa-field (from 1939 on called mesons), Kemmer ${ }^{25}$ found in 1938 that, assuming that the expression of the energy be positive definite, the

\footnotetext{
${ }^{20}$ I.e., transformable into an Einstein space by means of a conformal transformation.

${ }^{21} \mathrm{~J}$. A. Schouten and J. Haantjes, Proc. Kon. Akad. v. Wet. 39, 1059 (1936).

${ }^{22}$ J. Haantjes; Proc. Kon. Akad. v. Wet. 43, 1288 (1940).

${ }^{23}$ E. L. Hill, Phys. Rev. 72, 143 (1947).

${ }^{24}$ H. Yukawa, Proc. Phys. Math. Soc. Japan 17, 48 (1935)

${ }^{25}$ N. Kemmer, Proc. Camb. Phil. Soc. 34, 354 (1938).
}

wave function has the valence 1 or 2 , and that consequently there exist four different kinds of meson fields. A wave function of valence 2 is an affinor in $\widetilde{\Xi}_{4}$ with 16 components that splits into a (symmetrical) tensor with 10 and a bivector with 6 components. The tensor corresponds in $R_{4}$ to the combination of a vector or pseudovector (=trivector) and a bivector, and the bivector to the combination of a scalar, a vector, and a pseudoscalar (=quadrivector). The first combination is usually called a vector meson field and the second (dropping either the scalar or the pseudoscalar as a trivial solution) as a scalar meson field with 5 components. Now Möller and Rosenfeld ${ }^{26}$ found in 1940 that the experiments seemed to require a very special combination of a scalar and a vector meson field (the "mixed" theory) and in 1941 Möller $^{27}$ discovered that just this combination appeared in a very natural way, if 5 coordinates instead of 4 coordinates in space time were introduced. It is true that Jauch and $\mathrm{Hu}$ in 1944 pointed out that there exist several possible field mixtures and that probably the mixture of Möller and Rosenfeld was not the most profitable one, but this does not change the fact that it was proved once for all that by introducing a more general fundamental group (i.e., a more general geometry) relations between different kinds of fields could be fixed automatically. The interpretation of the 5 coordinates of Möller was not a projective one, but this did not have any influence on the results as was pointed out by Pais. ${ }^{28}$

Now the three possibilities, the scalar meson field, together with the trivial case with 6 components, the mixed case with 15 components and the vector meson field with 10 components remind one immediately of the numbers of components of the vector, the bivector and the half-trivector (that plays a fundamental role in the representation theory of rotations) in 6 dimensions, and following this hint, Lubanski and Rosenfeld ${ }^{29}$ could prove in 1942 that the typical equation of the matrices occurring in the wave equation of mesons is identical with the so-called structural relation of the group of rotations in $R_{6}$. Here is a table of the different possibilities, in which we neglected for the sake of simplicity the difference between ordinary quantities and pseudoquantities. We remind that a $p$-vector in $R_{n}$ splits always into a $p$-vector and a $(p-1)$-vector in $R_{n-1}$ (e.g., the electromagnetic bivector in $R_{4}$ into the magnetic bivector (pseudovector) and the electric vector in $R_{3}$ ). $\mathrm{Pais}^{28}$ remarked that in general projective geometry we always have at our disposition the projective vector $X^{K}$, corresponding to the "contact point" of local space and that by using this projective vector another scalar and another vector meson field

${ }^{26}$ C. Möller and L. Rosenfeld, Kgl. Danske Vid. Sels. Math.fys. Medd 17 (1940).

${ }^{27}$ C. Möller, Kgl. Danske Vid. Sels. Math.-fys. Medd 18 (1941).

${ }^{28}$ A. Pais, Physica 9, 267 (1942)

${ }^{29}$ J. K. Lubanski and L. Rosenfeld, Physica 9, 107 (1942). 


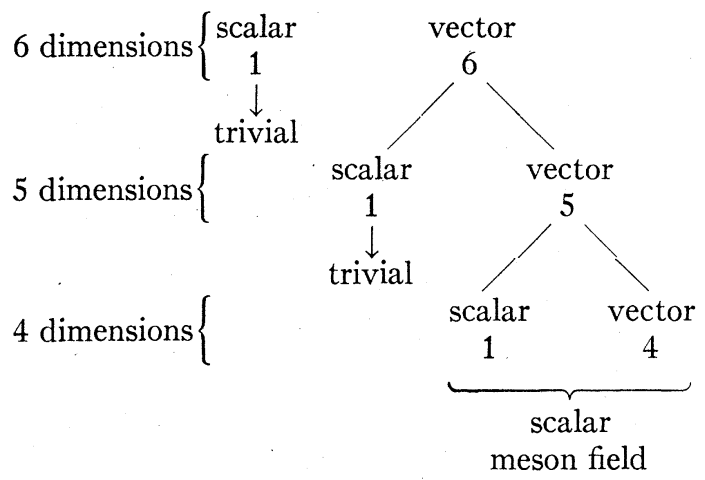

can be constructed from the mixed case. In this paper Pais gives the equations of all five possible meson fields.

That was the third time conformal theory came as an uninvited and quite unexpected guest. But the next time it got a real invitation. B. Hoffmann, induced by the 5-dimensional treatment of the meson field, again took up in $1947^{30}$ his former investigations with Veblen on projective relativity and tried to derive the field equations from a projective variation principle. The results being not quite satisfying he considered the possibility of a conformal treatment and developed this idea in 1948 in two papers. ${ }^{31}$ It is remarkable that his passing from projective to conformal geometry was only induced by the invariance of Cunningham and Bateman and by the conformal treatment of spin theory and not yet by the introduction of 6 dimensions in the paper of Lubanski and Rosenfeld. In fact, he mentions this introduction only in the second paper of 1948. Hoffmann does not use general conformal transformations but only such that change the fundamental tensor in all points with the same constant factor. His investigations are not closed, he himself calls them very modestly only an exploration, but that does not change the fact that in his papers for the first time the conformal character of the meson field was established explicitly, and the number 6 connected with its wave function was explained.

The investigations of $\mathrm{H}$. T. Flint are perhaps in some way connected with conformal meson theory though they arise from quite another line of thought. Like Yositaka Mimura he tried $^{32}(1935-37)$ to replace the length of the linear element by a matrix. From $1940 \mathrm{on}^{33}$ he uses 5 coordinates. Then he starts from a unified theory in 5 dimensions and introduces a meson field by means of a variable gauge-factor in the fundamental tensor in the same way as Weyl did in the 4-

${ }^{30}$ Banesh Hoffmann, Phys. Rev. 72, 458 (1947).

31 Banesh Hoffmann, Phys. Rev. 73, 30, 1042 (1948).

${ }^{32}$ H. T. Flint, Proc. Roy. Soc. 150, 421 (1935)

${ }^{33}$ H. T. Flint, Phil. Mag. 29, 330 (1940). dimensional theory. Hence, his meson field is in the same relation with gravitational-electromagnetic theory as the electromagnetic field is with gravitational theory. This introduction of a gauge-factor smells rather "conformal," and so we might expect that introduction of 6 coordinates into Flint's theory would lead to remarkable simplifications and perhaps to new results. Now there is one thing in Flint's theory that does not agree with conformal ideas and that is his looking for invariant masses and lengths, because, as we have seen, only a product of mass and length can be conformally invariant. However, a comparison of Flint's most interesting theories with other theories is very difficult because of his quite different definition of the linear element.

As a mathematician the author has tried to give an account of some interesting features of the mathematical side of the problem of conformal relativity in order to stimulate some physicists to investigations along these lines, using general homogeneous curvilinear coordinates and not only a subgroup of the conformal group but the whole group itself. But he has not tried to do any physics himself. Because he is convinced that this is a job only physicists can do properly. Only a physicist, using the results of experiments, is able to make the right choice from the overwhelming number of possibilities. A mathematician is too much interested in the beauty of his constructions and in mathematical physics this leads too often to formalism. There exist very few scientific men that are at the same time mathematician and physicist. For these children of the gods no rules exist, but as a matter of fact, ordinary mathematicians have seldom booked much success by meddling with physics, unless they succeeded in making some good physicist so very angry that he was stimulated to take over the job and finish it properly.

Our task as mathematicians is to do all the preliminary mathematical work, to make the mathematical instruments, to give advice in the use of them and to assist physicists in their work by throwing mathematical light if things grow too unsystematic or too complicated. 